\title{
ANN, SVM and KNN Classifiers for Prognosis of Cardiac Ischemia- A Comparison
}

\author{
H.S. Niranjana Murthy and Dr.M. Meenakshi
}

\begin{abstract}
This research work provides the comparison of performance of Artificial Neural Network (ANN), Support vector machine (SVM) and K-Nearest-Neighbour (KNN) models for cardiac ischemia classification. The novelty of this work is to develop an ANN, SVM and KNN model for classification of cardiac ischemia based on morphological changes in ECG signals. ST-depression, ST-elevation and Twave inversion changes in ECG signal are the early indicators of cardiac ischemia. Ischemic beats are classified from normal beats using morphological features extracted from ECG beat. The proposed ANN, SVM and KNN models receive the morphological features extracted from preprocessed ECG beat. The performance of all models are compared and validated on physiobank database in terms of accuracy, sensitivity and specificity. The results of this study exhibits that the proposed ANN based model has great potential than that of SVM and KNN classifiers for classification of cardiac ischemia.
\end{abstract}

Keywords--- Feature Extraction, Support Vector Machine, Artificial Neural Network, K-Nearest-Neighbour

\section{INTRODUCTION}

$\mathrm{T}$ HE estimate of World Health Organization (WHO) shows a statistics of 17.3 million deaths which have occurred worldwide due to the Cardiovascular disease (CVD) [1]. One of CVD risk factors is atherosclerosis which can be diagnosed by myocardial ischemia detection. Cardiac ischemia is a cardiovascular disorder which affects the heart and the blood vessels.The coronary arteries become constricted by atherosclerosis which restricts the flow of blood and oxygen to the heart and results in heart attack or heart stroke. Chest pains occur when the blood received by the heart muscles is insufficient [2]. Diagnosing the Cardiac ischemia takes more time if examined by doctor using long duration ECG data. So, there is a great scope for the development of automatic technique for early detection of ischemia.

Cardiac Ischemia is reflected in ECG as ST-segment deviation and $\mathrm{T}$ wave amplitude changes. Further the works by papaloukas et al. [3] on long duration ECGs confirmed that the solution to early diagnosis of myocardial ischemia lies in accurate detection of ST-segment deviation and T-wave changes. The automatic detection of characteristic features

H.S. Niranjana Murthy, Assistant Professor, Department of Instrumentation Technology, M.S.R.I.T, Bangalore, India. E-mail: hasnimurthy@rediffmail.com

Dr.M. Meenakshi, Professor, Department of Instrumentation Technology, Dr.A.I.T, Bangalore, India.E-mail:meenakshi_mbhat@yahoo.com from ECG waves plays an important role in diagnosing myocardial ischemia. Hamid Gholam Hosseini [4] showed that evaluation of the ST segment together with T-wave changes indicate that the zone of ischemia is around the applied lead. Therefore, analysis of the ST segment is an important task in cardiac ischemia diagnosis. Gu-Young Jeong et al. [5] proposed a ST shape classification in ECG by constructing reference ST set of an ECG for detecting myocardial ischemia. In this method, classification of STs was carried out by using morphological changes on the test ECG data and the global correct rate achieved was $83.14 \%$. Papaloukas et al. [6] developed a rule based expert system for distinguishing the ST segment deviation and T-wave alterations which showed predictive accuracy of $80.09 \%$ in detecting T-wave episodes. Further, the works of Ozbay.Y et al. demonstrated that taking samples as feature values in the intervals of R-R are very effective in representing the classes of ECG signals representing different arrhythmias [7]. The study by Niranjana Murthy et al. [8] showed that Daubechies wavelets are best suitable for detecting ischemic beats with good recognition rate.

In recent years, there has been an increasing interest in developing soft computing techniques for effective detection of cardiac ischemia. To overcome the mortality rate due to coronary heart disease, there are many techniques developed in the literature. One among them is artificial neural network (ANN) model developed by Niranjana Murthy et al. [9] to predict coronary heart disease based on risk factors comprising of ST-segment and T-wave amplitude changes. Guler et al. [10] adopted two stages of neural networks for classifying input ECG signal into four types of beats and to improve the diagnostic accuracy [10]. SVM is another class of machine learning algorithm that can perform pattern recognition based on the theory of statistical learning [11]. Jianxin Chen et al. [12] compared SVM, neural networks, Bayesian classifier, decision tree and logistic regression for classifying the coronary heart disease and the result showed that the SVM technique classified the disease with highest accuracy of $90.5 \%$. The KNN method is an instance based learning which is widely used data mining technique in pattern recognition and classification problems [13]. The study by Mai Shouman et al. [14] showed that a highest value of classification accuracy is obtainable by varying the values of parameter K. The ECG signals obtained from the physiobank database were classified. PhysioBank is a large and growing documentation of well-distinguishable digital recordings of physiologic signals and related data for use by the biomedical research community [15]. 
The majority of the earlier works considered only a single method which includes ANN or SVM or KNN for classification of cardiac ischemia. This limits the classification accuracy because of non-availability of comparison of performance with other methods. To mitigate this drawback, this paper proposes development and comparison of ANN, SVM and KNN classifier models to identify the best classifier for ischemia classification from ECG. In the present work, ECG signal is preprocessed and denoised followed by the feature extraction by capturing samples between R-R intervals. Next, these features are used as inputs for training and testing the ANN, SVM and KNN models. The main contribution of this work is the development of a MLP neural network, SVM and KNN model and determining the effect of number of hidden neurons, various kernel functions, parameter $\mathrm{K}$ and identification of best classifier model.

The organization of this paper is as follows. Section 2 presents the methodology adopted, preprocessing of ECG signal, morphological feature extraction and Classifiers. Next, performance evaluation and experimental results of ANN, SVM and KNN classifiers are highlighted in section 3. Finally, conclusions are drawn at the end in section 4 .

\section{Methodology}

\section{A. Proposed ANN and SVM Classification Model}

Fig.1 shows the block diagram representation of the steps involved in the proposed cardiac ischemia classification model. The ECG samples acquired from Physionet database were preprocessed and then morphological features were extracted that can be used as inputs for an ANN, SVM and KNN model for classification. The entire system can be broken down into three disjoint modules: preprocessing ECG signal, feature extraction and classification which are discussed in the following sections.

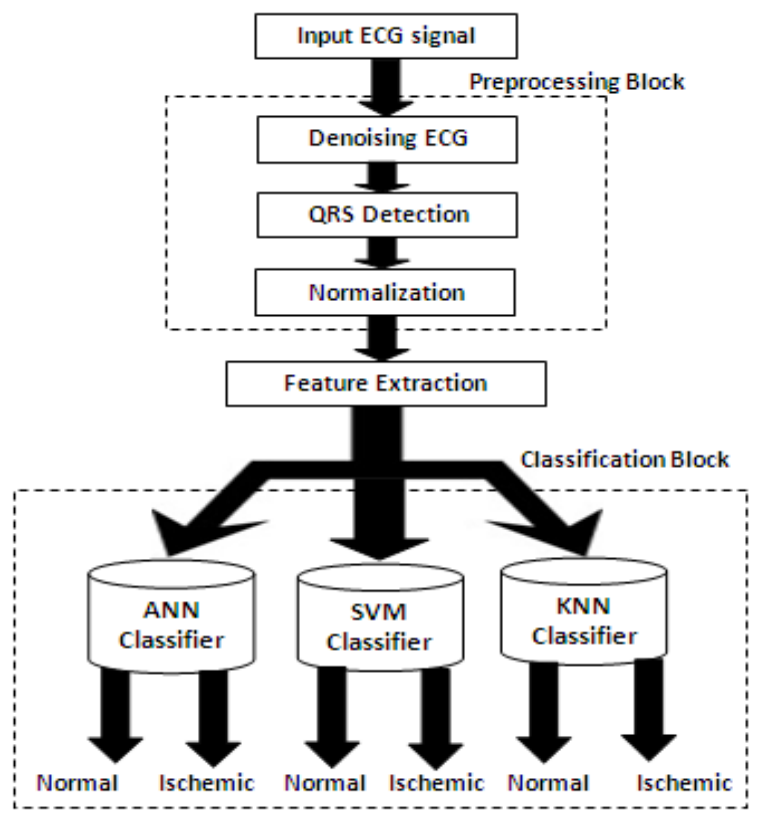

Figure 1: Block Diagram of Proposed Method

\section{B. Preprocessing of ECG Signal}

The ECG data acquired from physionet database may contain noise and needs to be preprocessed for removal of noise. The process of denoising ECG signal can be subdivided into four levels of filtering applied to ECG records; DC component removing, low pass filtering, high pass filtering and a comb filter. For removing unwanted dc component from ECG signal, the mean of the signal is subtracted from the signal itself and the baseline amplitude is pulled back to level zero.To remove high frequency random noise, generally caused by patients muscle contractions, a 10 point moving average low pass filter is used. The low frequency noise shows itself as baseline wandering that is caused generally by the respiration of the patient. For removing this low frequency noise, a derivative based high pass filter that cutoff the low frequencies is used. Power line interference is a noise caused by the electricity current flowing in wires and power lines which induces $60 \mathrm{~Hz}$ frequency component into ECG signal. To eliminate the power line interference, a band-stop comb filter which attenuates $60 \mathrm{~Hz}$ frequency component is used.

The QRS complex of ECG signal reflects the electrical activity within the heart during ventricular contraction. The time of its occurrence as well as its shape provides clinically useful information about the current state of heart. There are many different QRS detection techniques but this work uses Pan-Tompkins algorithm. The algorithm includes a sequence of modules that perform derivative, squaring, integration, adaptive threshold and search procedures. Finally, the negative values are removed from ECG signal and normalized between 0 and 1 for obtaining better results from an ANN, SVM and KNN model.

\section{Morphological Feature Extraction}

Selection of the inputs for ANN, SVM and KNN classifiers is the most important part of designing the classifiers models based on pattern classification. This is for the reason that even the best classifier will perform poorly if the inputs are not selected properly. Input selection has two meanings: (1) which components of a pattern, \& (2) which set of inputs best represent a given pattern. In this work, the QRS beats are selected at first. Secondly, a rectangular window which was formed by 150 discrete data was selected so that it contained clinical useful information between R-T intervals of ECG beat.

Literature demonstrates that taking samples between R-R intervals of ECG waves as feature values provides clinically useful information of the cardiac conditions. ST-depression, ST-elevation and T-wave inversion changes in ECG signal are the indicators for early detection of likelihood occurrence of myocardial ischemia. The denoised and normalized signal obtained in section 2.2 is used as the inputs for the feature extraction stages. From the results it is observed that each member of the particular class either diseased or normal shows the existence of similar patterns between R-R intervals. Hence, feature extraction is achieved by capturing 150 samples between R-R intervals which approximately correspond to R-T interval for ECG signal with sampling frequency of $250 \mathrm{~Hz}$. Training sets were formed by 297 vectors of 150 dimensions representing normal, ST depressed; ST 
elevated and $\mathrm{T}$ wave inverted class of beats. The test sets were also formed by 296 vectors of 150 dimensions. The term vector is used for defining the extracted features of the samples of an ECG beat.

\section{ANN, SVM and KNN Classifiers}

The general structure of a MLP network is shown in fig. 2 . MLPs are the simplest and most frequently chosen neural network architecture due to their configurationally flexibility, good representational capabilities and large number of programmable algorithms. The best ANN architecture is derived by trial and error and the complexity of the neural network is characterized by the number of hidden neurons.

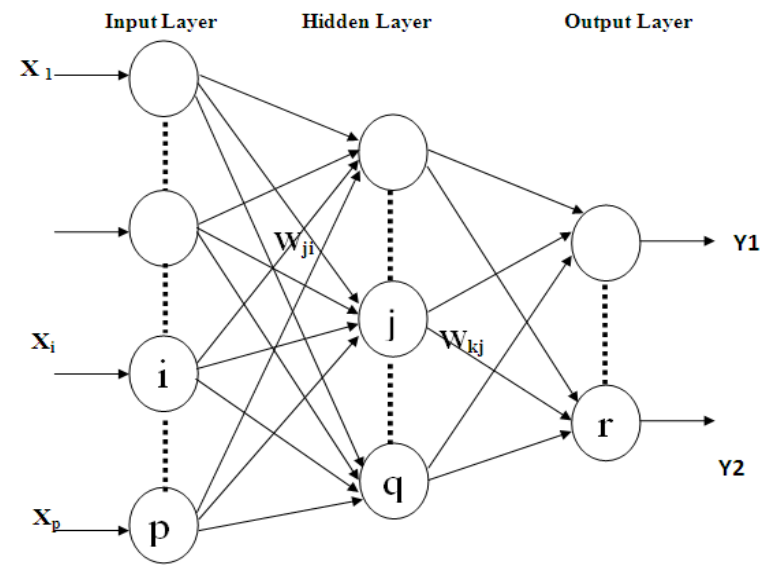

Figure 2: Structure of a MLP Network

The challenge in training phase of the MLP network is to decide the number of neurons in the hidden layer since there is no general rule available in the literature for selecting optimal number of hidden layer neurons. In the present work, the MLP network was trained by using the gradient descent algorithm with momentum and adaptive learning rate back propagation. In this work, the MLP neural network is implemented in MATLAB where the optimal number of hidden layer neurons is obtained by trial and error. After several trials, the results showed better performance with 48 hidden neurons. MLP neural network is implemented with sigmoid activation function.

The basic theory of an SVM is to map the input data onto a higher dimensional feature space nonlinearly related to the input space and determining a separating hyper plane with maximum margin between the two classes in the feature space [16]. Using SVM, an optimal separating hyper plane in the higher dimensional feature space can be computed by using kernel functions in the input space. In the proposed work, the experiment is carried out with SVM classifier and studied the effect of various kernel functions on classification accuracy, sensitivity and specificity.

KNN is a simple algorithm that classifies new cases based on a similarity measure. It is a simple non-parametric technique for classification. KNN classifier required a metric $\mathrm{d}$ and a positive integer $\mathrm{K}$ [17]. Boshra Bahrami et al. [18] have established that Euclidean distance metric is a simple and easy to implement method for computing distances in multidimensional input space which can yield competitive results even compared to the most sophisticated learning methods. KNN stores the position of training samples and their class. When the new testing data is fed, KNN computes the distance between the query data and training samples. Based on the defined threshold $\mathrm{K}$ number, $\mathrm{K}$ samples with least distances are selected and the class with more samples in the boundary is the result.

\section{RESUlTS \& DisCUSSIONS}

\section{A. Performance Measure}

The performance of the proposed model is evaluated by computing the percentages of Sensitivity (SE), Specificity (SP) and Correct Classification i.e. Accuracy (AC). These validation parameters are defined as:

$$
\begin{gathered}
\text { Sensitivity }(\mathrm{SE})=\frac{\mathrm{TP}}{\mathrm{TP}+\mathrm{FN}} \\
\text { Specificity }(\mathrm{SP})=\frac{\mathrm{TN}}{\mathrm{TN}+\mathrm{FP}} \\
\begin{array}{l}
\text { Classification } \\
\text { accuracy }(\mathrm{AC})=\frac{\mathrm{TP}+\mathrm{TN}}{\mathrm{TN}+\mathrm{FP}+\mathrm{TP}+\mathrm{FN}}
\end{array}
\end{gathered}
$$

Where TP (True Positive) is the number of subjects correctly classified as healthy, TN (True Negatives) is the number of subjects correctly classified as abnormal; FN (False Negatives) is the number of subjects misclassified as abnormal when actually normal, and FP (False Positives) is the number of subjects misclassified as normal when actually abnormal [19]. In the classification problems, the purpose of the network is to assign each case to one of the classes.

\section{B. Experimental Results}

Fig. (3) Shows the result of ECG signal denoising and QRS detection achieved by passing the ECG signal through different stages explained in section 2.2.
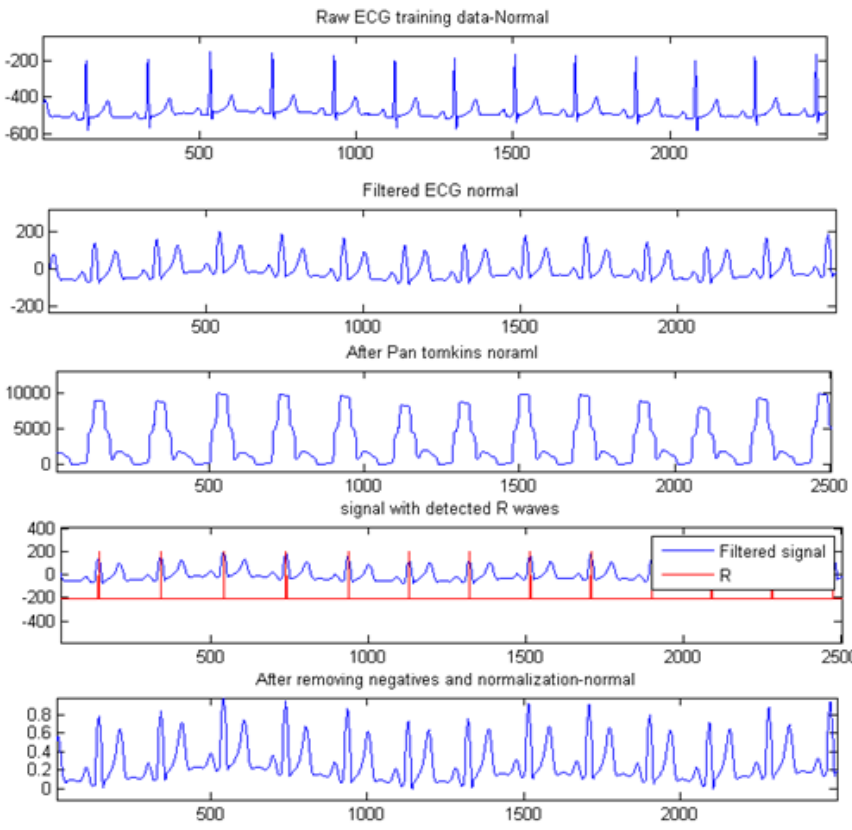

Figure 3: ECG signal Denoising \& Normalization Stages 
The feature vectors were calculated as explained in section 2.3. The satisfactory operation of neural network depends on the sizes of training set and test set. The 297 vectors of 150 dimensions were formed for training set. The 296 vectors of 150 dimensions were formed for test set. The term vector is used for defining the extracted features of the samples of an ECG beat.

The performance of the MLP neural network is evaluated by the computation of classification sensitivity, specificity and accuracy. The classification results indicated that the MLP neural network trained with the gradient descent algorithm with momentum and adaptive learning rate back propagation was efficient algorithm for detecting variability in the ECG signals. To choose best architecture, several architectures are constructed with varying number of hidden neurons for the network parameters given in table 1.

Fig.(4) shows the comparison of training and testing classification accuracy for MLP architecture with different numbers of hidden neurons. It is inferential from the chart that the MLP neural network model with 48 hidden neurons classifies the ischemic beats from normal beats with high accuracy. In this work, the MLP architecture with 48 hidden neurons has performed best with high values of training \& testing classification accuracy. The highest classification accuracy of SVM model is determined for each kernel function by varying its parameters. Similarly, the optimum performing KNN classifier model is found by varying the value of threshold $\mathrm{K}$ number. Table 2 shows the comparison of classification accuracy of ANN, SVM and KNN classifiers.

Table 1: Network Architecture Parameters

\begin{tabular}{|l|l|}
\hline Best Architecture: & {$[$ 150-48-4 ] } \\
\hline Iterations: & 205 \\
\hline Training Algorithm: & Gradient Descent Back propagation \\
\hline Minimum Error Goal: & 0.001 \\
\hline Learning Rate: & 0.01 \\
\hline Momentum: & 0.9 \\
\hline Activation: & Sigmoid \\
\hline Performance Criteria: & Mean squared Error \\
\hline
\end{tabular}

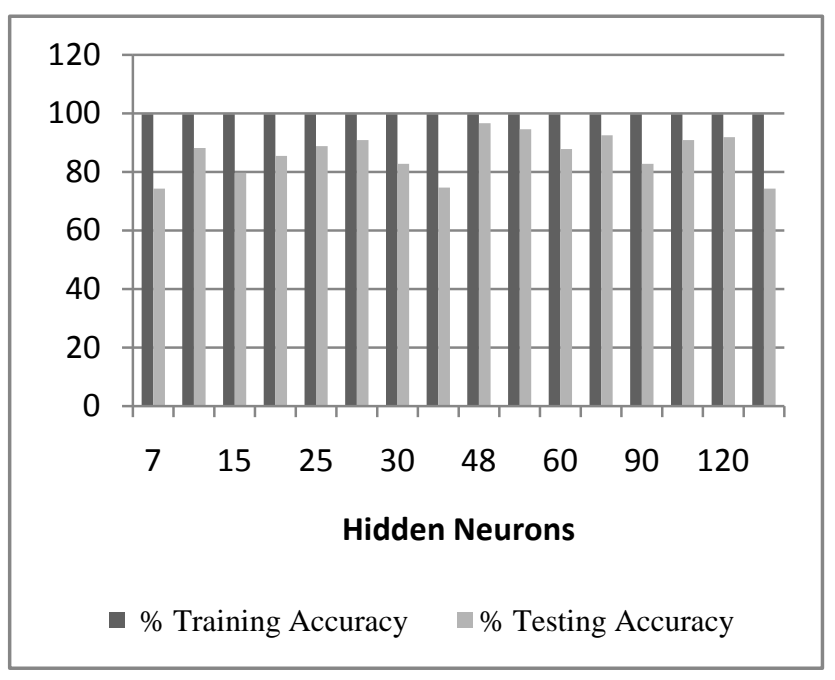

Figure 4: Variation of Classification Accuracy

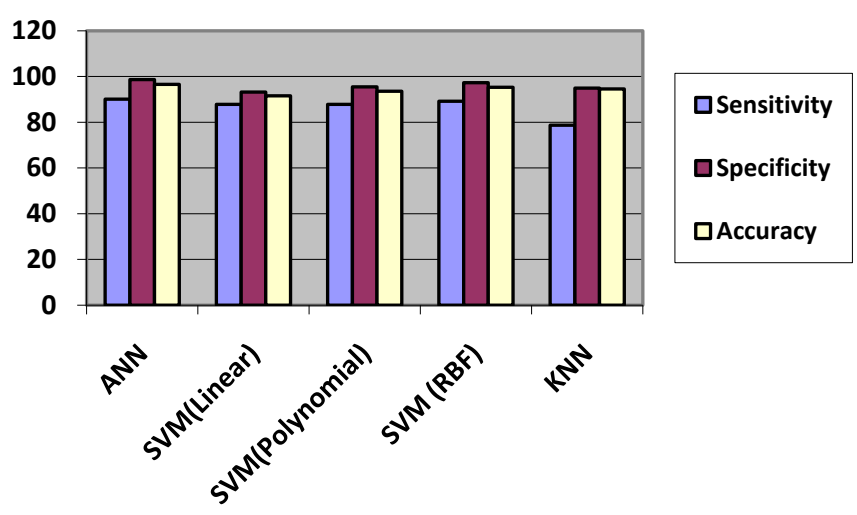

Figure 5: Performance Comparisons of ANN, SVM and KNN Classifier

Table 2: Network Architecture Parameters Comparison of Accuracy of ANN, SVM and KNN Methods

\begin{tabular}{|l|l|l|}
\hline Method & Kernel Type & Classification Accuracy \\
\hline MLP Neural Network & - & 96.62 \\
\hline Support Vector Machine & Linear & 91.55 \\
& Polynomial & 93.58 \\
& RBF sigma & 95.27 \\
\hline KNN Classifier & - & 94.59 \\
\hline
\end{tabular}

From the comparison of classifiers depicted in table.2, it is evident that the MLP neural network outperformed with highest value of classification accuracy. The result of this work shows that the network has successfully learnt and able to classify the new and unknown data into normal, ST depressed, ST elevated and T wave inverted.

\section{CONCLUSION}

This paper gives the comparison of ANN, SVM and KNN classifiers for cardiac ischemia classification based on morphological features extracted from ECG. In this study, experiment is conducted by varying the number hidden neurons in ANN, applying various kernel functions in SVM and changing the value of threshold $\mathrm{K}$ number in KNN classifiers to evaluate the best performance. The experimental results confirmed that the ANN model outperformed with testing classification accuracy of $96.62 \%$. This accuracy obtained is considerably high in comparison with SVM and KNN classifiers. Also, the work clearly indicates the improved accuracy by combining ANN with morphological features in early prediction of myocardial ischemia. A further analysis is required for optimal selection of the morphological features to achieve improved accuracy.

\section{ACKNOWLEDGEMENT}

Our thanks to the experts who have contributed in development of European ST-T datasets of physionet database. 


\section{REFERENCES}

[1] McGovern PG, Pankow JS, Shahar E, Doliszny KM, Folsom AR, Blackburn $\mathrm{H}$ and Luepker RV, "Recent trends in acute coronary heart disease: mortality, morbidity, medical care, and risk factors.” N Engl J Med. Vol. 334, pp. 884-890, 1996.

[2 ] Chen, J. and Greiner.R., "Comparing Bayesian Network Classifiers", proceedings of UAI-99, pp.101-108, 1999..

[3 ] C.Papaloukas, D. Fotiadis, A. Likas and L. Michalis, "Automated methods for ischemia detection in long duration ECGs”, Cardiovasc Rev Rep, vol.24, no.6, pp. 313-320, 2003.

[4 ] Hamid Gholam Hosseini, "Computer-aided diagnosis of cardiac events", Flinders University of South Australia, School of Informatics and Engineering, 2001.

[5] Gu-Young Jeong, Myoung Jong Yoon and EijiInooka, "ST shape classification in ECG by constructing reference ST set", Medical Engineering and physics, Vol.32, No.9, pp.1025-1031, 2010.

[6 ] Papaloukas.C, Fotiadis D.I. and Likas. A, "Use of a novel rule-based expert system in the detection of changes in the ST segment and the T wave in long duration ECGs", Journal of Electro cardiology, Vol. 35, No.1, pp.27-34, 2002.

[7] Ozbay.Y and Karlik.B, "A recognition of ECG arrhythmias using artificial neural network", proceedings of the $23^{\text {rd }}$ annual conferenceIEEE/EMBS, Istanbul,Turkey.O, 2001.

[8 ] H.S.Niranjana Murthy, M.Meenakshi, "ECG signal Denoising and Ischemic Event Feature extraction using Daubechies Wavelets”, Int. Journal of Computer Applications, Vol. 67-2, 2013.

[9 ] H.S.Niranjana Murthy, Meenakshi. M, "ANN model to predict coronary heart disease based on risk factors", Bonfring Int. J. Man Mach Interface, volume 3, No.2, June 2013.

[10 ] Guler.I and Ubeyli.E, "ECG beat classifier designed by combined neural network mode”, Pattern Recognition, Vol. 38, pp.199-208, 2005.

[11 ] Idicula-Thomas. S,.Kulkarni, A.J, Kulkarni B.D, Jayaraman.V.K, and Balaji.P.V, "A SVM based method for predicting propensity of a protein to be soluble or to form inclusion body on over expression in Escherichia coli”, Bioinformatics, 2006.

[12 ] Jianxin Chen, Guangcheng Xi, Yanwei Sing, Jing Chen and Jie wang, “ Predicting Syndrome by NEI Specifications: A comparison of Five Data Mining Algorithms in Coronary Heart Disease”, Life System Modeling and Simulation Lecture Notes in Computer Science, pp 129-135.

[13 ] F.Moreno-Seco, L.Mico and J.A.Oncina, "Modification of the LAESA Algorithm for Approximated k-NN Classification”, Pattern Recognition Letters, pp.47-53, 2003

[14 ] Mai Shouman, Tim Turmer and Rob Stocker, “ Applying k-Nearest Neighbor in Diagnosing Heart Disease Patients”, International Conference on Knowledge Discovery, IACSIT press, Singapore, 2012.

[15 ] Goldberger. A.L.,Amaral, L.A.N, Glass.L, Hausdorff.J.M, Ivanov.P.Ch. and Mark.R.G, Physiobank, "Physiotoolkit and Physionet: Components of a new research resource for complex physiologic signals", Circulation, 101(23), e215-e220, June 13, 2000.

[16 ] C.J.C.Burges, “A tutorial on SVM for pattern recognition”, Data Mining and Knowledge Discovery, pp 121-167, 1998.

[17 ] Indu Saini, Dilbag Singh and Arun Khosla, "QRS detection using KNearest Neighbor algorithm and evaluation on standard ECG databases", Journal of Advanced Research, pp.331-344, 2013.

[18 ] Boshra Bahrami and Mirsaeid Hosseini Shirvani, "Prediction and Diagnosis of Heart Disease using Data Mining Techniques", JMEST, ISSN: 3159-0040, Vol. 2, Issue 2, Feb. 2015

[19 ] Hagan, M.T. and Menhaj, M.B., "Training Feedforward Networks with the Marquardt Algorithm”, IEEE Transactions on Neural Networks, Volume: 5, Issue 6, Nov 1994.

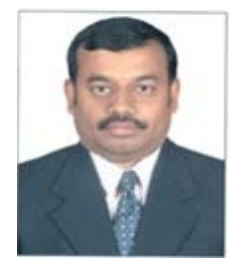

H.S.Niranjana Murthy was born in Karnataka, India on July 29, 1974 . He received his B.E. degree in Instrumentation \& Electronic Engineering from Bangalore University, Karnataka, in 1996. He received M.Tech degree in Computer applications in Industrial drives from Mysore University, Karnataka, in 1999. He is currently working as Assistant Professor (Sr. grade), Department of Instrumentation Technology, M.S.Ramaiah Institute of Technology, Bangalore. He has more than 14 years of experience in teaching. He is presently research scholar in Visvesvaraya Technological University (VTU). His research interests are Artificial Intelligence, Control systems, Biomedical Instrumentation. He has presented many papers in national conferences \& published papers in International journals. He is a Life member of MISTE. He is a member of BOS of Dept. of Instrumentation Technology, MSRIT, autonomous institute affiliated to VTU. He is also the Faculty Advisor for ISA Student section, MSRIT. (E-mail: hasnimurthy@rediffmail.com)

Dr.M. Meenakshi is graduated from SJCE Mysore in the field of Instrumentation Technology. She received her Master Degree in the field of Controls, Guidance and Instrumentation from I.I.T Madras and Ph.d degree from the department of Aerospace engineering. I.I.Sc Bangalore in the field of Controls \& Instrumentation. She has a teaching experience of 20 years. She published more than 40 research publications, including International Journals, International Conferences, National Conferences, workshops and seminars. She is currently Professor \& Head of the Dep. Of Instrumentation Technology, Dr.AIT, Bangalore-56. (E-mail:meenakshi_mbhat@yahoo.com) 